\title{
Injury in China: a systematic review of injury surveillance studies conducted in Chinese hospital emergency departments
}

\author{
Michael Fitzharris ${ }^{1,2^{*}}$, James $\mathrm{u}^{3}$, Naomi Hammond ${ }^{2,3}$, Colman Taylor ${ }^{2}$, Yangfeng Wu ${ }^{4,5}$, Simon Finfer ${ }^{2,6}$ and
} John Myburgh'2,7,8

\begin{abstract}
Background: Injuries represent a significant and growing public health concern in China. This Review was conducted to document the characteristics of injured patients presenting to the emergency department of Chinese hospitals and to assess of the nature of information collected and reported in published surveillance studies.

Methods: A systematic search of MEDLINE and China Academic Journals supplemented with a hand search of journals was performed. Studies published in the period 1997 to 2007 were included and research published in Chinese was the focus. Search terms included emergency, injury, medical care.

Results: Of the 268 studies identified, 13 were injury surveillance studies set in the emergency department. Nine were collaborative studies of which eight were prospective studies. Of the five single centre studies only one was of a prospective design. Transport, falls and industrial injuries were common mechanisms of injury. Study strengths were large patient sample sizes and for the collaborative studies a large number of participating hospitals. There was however limited use of internationally recognised injury classification and severity coding indices.

Conclusion: Despite the limited number of studies identified, the scope of each highlights the willingness and the capacity to conduct surveillance studies in the emergency department. This Review highlights the need for the adoption of standardized injury coding indices in the collection and reporting of patient health data. While high level injury surveillance systems focus on population-based priority setting, this Review demonstrates the need to establish an internationally comparable trauma registry that would permit monitoring of the trauma system and would by extension facilitate the optimal care of the injured patient through the development of informed quality assurance programs and the implementation of evidence-based health policy.
\end{abstract}

\section{Background}

The magnitude of injury-related mortality and morbidity in China was highlighted by Wang and colleagues in the Health System Reform in China Series featured in The Lancet[1]. Wang et al. reported that $10 \%$ of all deaths and $30 \%$ of all potentially productive life years lost (PPYLL) were due to injury related causes. In numeric terms, this equates to the loss of approximately 850,000 lives per annum, with two-thirds of those killed being

\footnotetext{
* Correspondence: Michael.Fitzharris@monash.edu

${ }^{1}$ Accident Research Centre and Injury Outcomes Research Unit, Monash

Injury Research Institute, Monash University, Victoria, Australia

Full list of author information is available at the end of the article
}

less than 45 years of age [1]. Traffic-related injury (32.3\%), drowning (13.4\%), falls (9.7\%) and poisoning (4.5\%) were the leading causes of unintentional injury deaths, while suicide was the leading cause of intentional injury and the second leading cause of injury deaths (23\%) overall. Injuries represent the leading cause of death for persons under 40 years of age [2,3].

With close to 23 million disability-adjusted life years (DALYs) lost per annum (11.5\% of all-cause DALYs), unintentional injuries represent a significant source of morbidity. Road traffic crashes account for one-third of these DALYs, followed by 'other unspecified causes' (29\%), falls (17\%), drowning (15\%) and poisonings (6\%)

\section{Biomed Central}


[4]. An estimated 200 million persons are injured each year, with approximately one-third (62 million) requiring emergency care or hospitalisation [5]. The consumption of health resources as a consequence of injury is significant. Direct medical costs have been estimated to be as high as CNY 64.1 billion RMB (USD $\$ 9.3$ billion) per annum, with costs related to delay and absence from work being approximately CNY 6 billion (USD $\$ 0.8$ billion)[5], equivalent to $1.92 \%$ of GDP (2007) [6].

Within this context of high injury rates and perceived limited available epidemiological data, commentators have identified the need for the establishment of population based injury surveillance systems to guide public health programs $[3,7,8]$. A number of fatality reporting systems and data sources do however exist, these being the National Statistics Yearbook, the Transportation Statistics Yearbook, and the Health Statistic Yearbook, the latter which reports mortality statistics for select causes of injury. While cause-of-death data leads to an understanding of changing disease patterns and permits population health policy planning, hospital-based injury surveillance systems and trauma registries facilitate prevention efforts as well as forming the basis of hospital quality assurance programs [9]. It has been noted that to date such systems have been limited in their scope within China $[3,10,11]$.

Given the high incidence of injury in China and the calls for the establishment broad based injury surveillance programs, it was considered timely to document the extent to which injury surveillance studies have been conducted. Whilst also documenting the incidence and causes of injury for a wider audience, this Review aims to document existing research strengths as well as areas of surveillance systems research that require strengthening. Of particular interest was the extent to which the reporting of patient injury data is consistent with commonly accepted global reporting guidelines, and whether there is a need for broadbased injury surveillance and/or trauma registry systems to be implemented. In conducting this Review, there were two specific objectives:

1 . To describe the characteristics of persons presenting to an emergency department following injury and the associated mechanisms of injury, and

2. To document that type of patient and injury information commonly reported, and following this, determine the extent to which this reporting is consistent with commonly accepted global guidelines.

\section{Methods}

\section{Protocol and registration}

This systematic Review has not been registered. The research objectives, analysis methods and inclusion criteria are fully specified here.

\section{Study eligibility criteria}

Retrospective and prospective studies of injured persons presenting to an emergency department in China were the focus of this Review. Studies that included all-cause injury presentations published from 1997 to 2007 in the Chinese language were included. Studies that focused exclusively on traffic crashes, age cohort subsets or specific injuries were excluded from the Review.

\section{Information sources}

Studies were identified using electronic databases, a hand-search of the Tables of contents pages of general and specialist medical Chinese language journals (Table 1 ), and by scanning reference lists of identified articles. The initial search strategy included both Chinese and English language articles within the limits specified above, with 'Medline' and 'China Academic Journals Full-Text Database' used. The last search was performed in 11 July 2009.

\section{Search strategy}

For the computerised searches the following search terms were used: 'China'; 'emergency medical services'; emergencies; emergency; ambulances; air ambulance(s); 'emergency service, hospital'; 'emergency department'; 'pre-hospital care'; 'wounds and injuries'; accident(s). Multiple searches (4) were conducted and duplicate articles were identified and eliminated. The same search terms and strategy was used in both electronic databases (Table 2 appendix).

\section{Table 1 List of Journal outlets searched by hand}

\begin{tabular}{l}
\hline Chinese Journal of Epidemiology \\
\hline Chinese Journal of Critical Care; \\
\hline Chinese Journal of Traumatology \\
\hline Chinese Journal of Industrial Hygiene and Occupational Medicine \\
\hline Chinese Critical Care Medicine \\
\hline Journal of the Fourth Military Medical University \\
\hline Journal of Sichuan University (Medical Science Edition) \\
\hline Chinese Journal of Hospital Administration \\
\hline Chinese Journal of Geriatrics \\
\hline Chinese Hospital Management \\
\hline Journal of Tongji University (Medical Science) \\
\hline Journal of Traumatic Surgery \\
\hline Shanghai Journal of Preventative Medicine \\
\hline Orthopaedic Journal of China \\
\hline Chinese Journal of Emergency Medicine
\end{tabular}


Table 2 Appendix - SEARCH STRATEGY - MEDLINE (OVID)

\begin{tabular}{|c|c|}
\hline $\begin{array}{l}\text { Search } \\
1\end{array}$ & \\
\hline 01 & China.mp or China/ \\
\hline 02 & $\begin{array}{l}\text { Emergency Medical Services or emergency medical services. } \\
\mathrm{mp}\end{array}$ \\
\hline 03 & Emergencies or emergencies.mp \\
\hline 04 & 2 or 3 \\
\hline 05 & 1 and 4 \\
\hline 06 & limit 5 to humans \\
\hline 07 & limit 6 to Chinese \\
\hline \multicolumn{2}{|l|}{$\begin{array}{l}\text { Search } \\
2\end{array}$} \\
\hline 01 & China.mp or China/ \\
\hline 02 & $\begin{array}{l}\text { Ambulances or Air Ambulances or ambulance.mp or pre- } \\
\text { hospital care.mp }\end{array}$ \\
\hline 03 & 1 and 2 \\
\hline 04 & limit 3 to humans \\
\hline 05 & limit 4 to Chinese \\
\hline \multicolumn{2}{|l|}{$\begin{array}{l}\text { Search } \\
3\end{array}$} \\
\hline 01 & China.mp or China/ \\
\hline 02 & Emergency Service, Hospital or emergency department.mp. \\
\hline 03 & 1 and 2 \\
\hline 04 & limit 3 to humans \\
\hline 05 & limit 4 to Chinese \\
\hline \multicolumn{2}{|l|}{$\begin{array}{l}\text { Search } \\
4\end{array}$} \\
\hline 01 & China.mp or China/ \\
\hline 02 & injury.mp. or "Wounds and Injuries"/ \\
\hline 03 & accident.mp. or Accidents/ \\
\hline 04 & 2 or 3 \\
\hline 05 & 1 and 4 \\
\hline 06 & limit 5 to humans \\
\hline 07 & limit 6 to Chinese \\
\hline
\end{tabular}

\section{Study selection and classification of the identified research}

One Review author (MF) conducted the searches and with the assistance of Review Author JY classified each study according to its principal focus. A classification scheme was developed (refer Additional file 1 Table S1) with the number of published papers in each category noted (refer Additional file 2 Table S2). Figure 1 presents the identification, screening, eligibility assessment and included studies in accordance with the PRISMA specification [12].

\section{Data items of interest}

In seeking to fulfill the second aim of this Review, the patient characteristics, injury severity and outcome indicator data fields of interest were specified a-priori. As the primary interest was in determining the comparability of reported data with internationally recognized best practice, the fields of interest were motivated by reference to the Utstein Style 'Recommendations for uniform reporting of data following major trauma' [13] and its subsequent revision [14], the Australia and New Zealand National Trauma Registry [15] and the American College of Surgeons National Trauma Databank [16] and Resources of Optimal Care of the Injured Patient[9]. The identified data fields are presented in Table 3 and each study included in the Review is compared across these data fields. In this Review it was considered too complex to include all data points from the above four reference documents; rather the items selected were done so on the basis of being the minimum key parameters required for comparisons across international studies. Particular attention was paid to whether studies reported the Abbreviated Injury Scale [17], the Injury Severity Score (ISS) [18], ICD codes [19], the Glasgow Coma Score [20], the Revised Trauma Score [21] and the Trauma Injury Severity Score (TRISS) [22].

\section{Data collection process}

Using the a-priori identified data items of interest data was entered into a MS Excel Spreadsheet for the 13 relevant studies. One author (MF) performed the initial data extraction which was verified by Author JY. Review author YW further resolved questions of interpretation from Chinese to English in the source articles.

\section{Results}

Thirteen research papers were identified that met the Review inclusion criteria [23-35]. The three search strategies identified 273 scientific papers, of which 143 were identified from Medline, 76 via the manual hand search and 54 from Chinese Academic Journals database. There were 268 unique papers following exclusion of five identified duplicate papers with 65 being hospital-based studies; of these, 13 were injury surveillance studies based in the emergency department (Table 4 Figure 1).

\section{Description of the identified studies: patient characteristics and injury mechanisms}

The 13 emergency department injury surveillance studies (nine prospective; four retrospective) were grouped into four categories: 1 . the ' 25 emergency department's studies'; 2. Prospective studies using the National Injury Surveillance System (NISS) Reporting Card; 3. Collaborative studies, and 4 . Single centre studies. Table 5 details the key aspects of each study and highlights the type of patient information collected. A brief description of each study is presented below both to provide the context for a discussion on the type of patient data collected and to fulfil Aim 1 of increasing the accessibility 


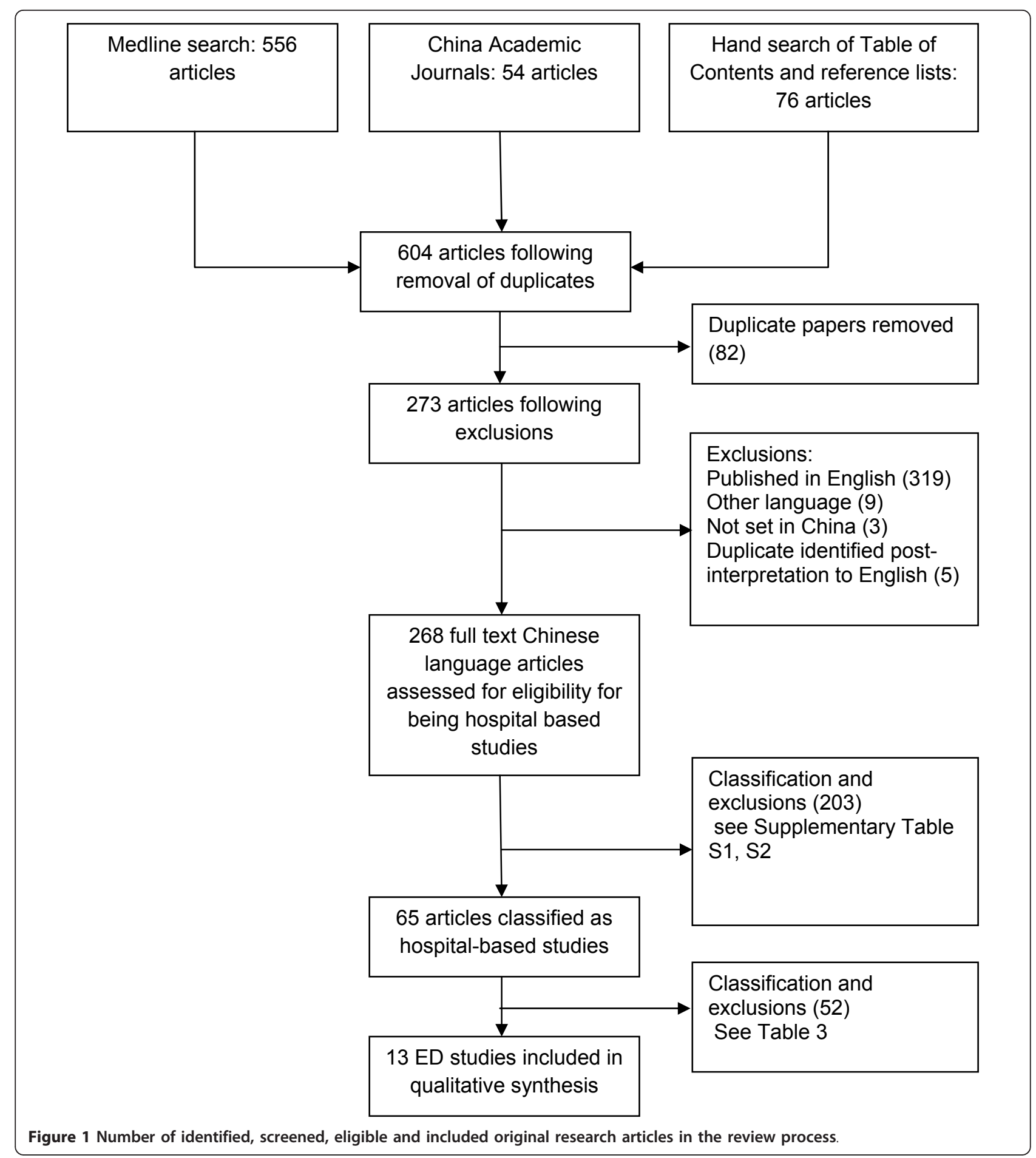

of Chinese injury surveillance research; in the main, the data discussed below is not presented in the Tables.

The '25 emergency departments' study

The '25 emergency departments' study aimed to determine the type of patients attending hospital due to injury, to report the mode of transportation to hospital, and to document mortality outcomes. This study was reported in two papers [23,24].

As a way of examining the feasibility of establishing a hospital-based injury surveillance system, Chen et al implemented a 'uniform survey' proforma to prospectively collect patient data in 25 hospitals [23]. The 'census' dates were the $1^{\text {st }}-15^{\text {th }}$ in each of July 2001, October 2001, 
Table 3 A-priori identified patient characteristic, injury severity and outcome indicator data fields of interest

\begin{tabular}{ll}
\hline Study design & Factors related to the circumstances of injury \\
\hline Design (prospective, retrospective) & Injury mechanism (transport - detail, fall, assault, poisons, burns etc...) \\
\hline Setting (location) & Location of injury (road, industrial, home) \\
\hline Number of hospital ED's in study & Injury coding and clinical indicators \\
\hline Data collection source/Survey tool (registry, survey) & Abbreviated Injury Score (AIS) [17] \\
\hline Date of study & Injury Severity Score (ISS) [18] \\
\hline Sample size & International Statistical Classification of Diseases and Health Related Problems (ICD) \\
\hline Patient factors & coding [19] \\
\hline Age & Glasgow Coma Score (GCS) [20] \\
\hline Gender & Revised Trauma Score (RTS) [21] \\
\hline Occupation & Trauma Injury Severity Score (TRISS) [22] \\
\hline System factors & Outcomes \\
\hline Pre-hospital care/mode of arrival & Admission to ICU \\
\hline Presentation/admission to ED (inclusion criteria of & Mortality rate \\
\hline Review) & Length of stay \\
\hline
\end{tabular}

January 2002 and April 2002 (Table 5). In the 60 day period, 143,274 patients presented to ED of which 25,019 (17.4\%) patients presented due to injury. Of these, $91.4 \%$ were described as having sustained 'acute injury' and 8.6\% as 'poisoning'. The overall injury mortality rate was $0.5 \%$ although mortality was higher for poisonings (1.1\%) than for acute injury $(0.4 \%)$ patients. The leading cause of injury was reported as 'mechanical injury' in the industrial and farming context (32.7\%) followed by traffic crashes $(26.9 \%$,

Table 4 Article sub-types for hospital-based injury studies

\begin{tabular}{ll}
\hline Focus of article & Number \\
\hline Emergency Department injury surveillance & 13 \\
\hline Inpatient injury surveillance & 3 \\
\hline Management of injury, practices & 8 \\
\hline Injury secondary to disease & 0 \\
\hline Body region specific injury & 4 \\
\hline Traumatic brain injury & 4 \\
\hline Face & 3 \\
\hline Thorco-abdominal & 2 \\
\hline Extremities & 1 \\
\hline Multi-trauma & 1 \\
\hline Age group specific & 11 \\
\hline Paediatrics & 6 \\
\hline Child & 6 \\
\hline Older adults & 3 \\
\hline Retrospective prediction mortality model & 65 \\
\hline Cause specific & \\
\hline Poisoning & \\
\hline Total & \\
\hline
\end{tabular}

6147). Traffic crashes accounted for nearly $47 \%$ of deaths. The male to female ratio was 2:1 for age groups under 60, above which the ratio was $1.07: 1$. Only $14.4 \%$ were transported to the emergency department by emergency vehicle with the remainder described as 'other means' or 'private'.

Using the same data, $\mathrm{Li}$ et al reported that injuryrelated admissions were higher in the 11 rural hospitals (29\%) compared to the 14 city hospitals (19\%), as was the mortality rate (rural: $1.29 \%$; city $0.27 \%$ )[24]. Transport accounted for $35 \%$ of injuries in rural hospitals followed by industrial machine type injuries (18.15\%), whereas the reverse was true for city hospitals (industrial machine type injuries: $33 \%$; transport: $21.8 \%$ ). The study collected and reported upon employment status, one of only three in this Review to do so (Table 6). Transportation workers (22\%, 74\% male) and students $(12.7 \%, 60 \%$ male) were the leading occupations in the city cohort, while in the rural hospitals farmers (37\%, $72 \%$ male), students $(14 \%$, $74 \%$ male) and transport workers (9\%, 87\% male) were the leading occupations. Mortality was the only clinical outcome variable reported in the study.

Reference to the a-priori established indicators of interest (Table 3 Table 5) highlights that no injury coding or clinical indicators were collected and reported in this study program. Despite this, the study was successful in establishing a comprehensive network that could serve as the basis for more detailed injury surveillance or integrated trauma registry systems.

\section{Prospective Studies using the National Injury Surveillance System Reporting Card}

Four studies [25-28] that utilised the Chinese-Centre of Disease Control (C-CDC) NISS Reporting Card [36] 
Table $\mathbf{5}$ Summary of key study characteristics

\begin{tabular}{|c|c|c|c|c|c|c|c|c|c|c|c|c|c|c|}
\hline & $\begin{array}{l}25 \mathrm{ED} \\
\text { study }\end{array}$ & $\begin{array}{l}25 \text { ED } \\
\text { study }\end{array}$ & $\begin{array}{l}25 \text { ED } \\
\text { study }\end{array}$ & $\begin{array}{l}\text { NISS RC } \\
\text { study }\end{array}$ & $\begin{array}{l}\text { NISS RC } \\
\text { study }\end{array}$ & $\begin{array}{l}\text { NISS RC } \\
\text { study }\end{array}$ & $\begin{array}{l}\text { NISS RC } \\
\text { study }\end{array}$ & $\begin{array}{l}\text { Collab. } \\
\text { Study }\end{array}$ & $\begin{array}{l}\text { Collab } \\
\text { study }\end{array}$ & $\begin{array}{l}\text { Single } \\
\text { centre }\end{array}$ & $\begin{array}{l}\text { Single } \\
\text { centre }\end{array}$ & $\begin{array}{l}\text { Single } \\
\text { centre }\end{array}$ & $\begin{array}{l}\text { Single } \\
\text { centre }\end{array}$ & $\begin{array}{l}\text { Single } \\
\text { centre }\end{array}$ \\
\hline & $\begin{array}{l}\text { Chen et } \\
\text { al [23] }\end{array}$ & $\begin{array}{l}\text { Li et al } \\
\text { [24] } \\
\text { City }\end{array}$ & $\begin{array}{l}\text { Li et al } \\
\text { [24] } \\
\text { Rural }\end{array}$ & $\begin{array}{l}\text { Zhang \& } \\
\text { Zhan [25] }\end{array}$ & $\begin{array}{l}\text { Zhou et } \\
\text { al [26] }\end{array}$ & $\begin{array}{l}\text { Xu et al } \\
\text { [27] }\end{array}$ & Li et al [28] & $\begin{array}{l}\text { Li \& } \\
\text { Wang } \\
\text { [29] }\end{array}$ & Li et al [30] & Li et al [31] & $\begin{array}{l}\text { Qu et al } \\
\text { [32] }\end{array}$ & $\begin{array}{l}\text { Zhou et al } \\
\text { [33] }\end{array}$ & $\begin{array}{l}\text { Yang et al } \\
{[34]}\end{array}$ & $\begin{array}{l}\text { Wen et } \\
\text { al [35] }\end{array}$ \\
\hline Design & Prosp. & Prosp. & Prosp. & Prosp. & Prosp. & Prosp. & Prosp. & Retro. & Prosp. & Prosp. & Retro. & Retro. & Retro. & $\begin{array}{l}\text { Retro. } \\
\text { (pre/post) }\end{array}$ \\
\hline $\begin{array}{l}\text { Setting/ } \\
\text { location }\end{array}$ & $\begin{array}{l}\text { Multiple } \\
\text { Provinces }\end{array}$ & $\begin{array}{l}\text { Multiple } \\
\text { Provinces }\end{array}$ & $\begin{array}{l}\text { Multiple } \\
\text { Provinces }\end{array}$ & $\begin{array}{l}\text { Qing-dao, } \\
\text { Shangdong }\end{array}$ & Henan & Guangdong & $\begin{array}{l}\text { Gaocheng, } \\
\text { He Bei }\end{array}$ & $\begin{array}{l}\text { Guang- } \\
\text { dong }\end{array}$ & $\begin{array}{l}\text { Shantou, } \\
\text { Guang- } \\
\text { dong }\end{array}$ & $\begin{array}{l}\text { Shantou, } \\
\text { Guang-- } \\
\text { dong }\end{array}$ & $\begin{array}{l}\text { Hangzhou, } \\
\text { Zhejiang }\end{array}$ & $\begin{array}{l}\text { Guangzhou, } \\
\text { Guangdong }\end{array}$ & $\begin{array}{l}\text { Guangzhou, } \\
\text { Guangdong }\end{array}$ & Chonqing \\
\hline $\begin{array}{l}\text { Number } \\
\text { Hospital }\end{array}$ & 25 & 14 & 11 & 6 & 3 & 10 & 26 & 332 & 2 & 1 & 1 & 1 & 1 & 1 \\
\hline Sample & 25,019 & 19,906 & 5113 & 1882 & 6948 & 42,567 & 7065 & $1,093,233$ & 2611 & 11,472 & 13,008 & 10,654 & 5346 & 8271 \\
\hline $\begin{array}{l}\text { Survey } \\
\text { tool }\end{array}$ & $\begin{array}{l}\text { Uniform } \\
\text { survey }\end{array}$ & see [23] & see [23] & $\begin{array}{l}\text { National } \\
\text { CDC RC }\end{array}$ & $\begin{array}{l}\text { National } \\
\text { CDC RC }\end{array}$ & $\begin{array}{l}\text { National } \\
\text { CDC RC }\end{array}$ & $\begin{array}{l}\text { National } \\
\text { CDC RC }\end{array}$ & $\begin{array}{l}\text { Report } \\
\text { form }\end{array}$ & $\begin{array}{l}\text { Uniform } \\
\text { survey }\end{array}$ & $\begin{array}{l}\text { Uniform } \\
\text { survey }\end{array}$ & $\begin{array}{l}\text { Registry } \\
\text { log }\end{array}$ & Not stated & Registry log & $\begin{array}{l}\text { Uniform } \\
\text { survey }\end{array}$ \\
\hline $\begin{array}{l}\text { Date of } \\
\text { study }\end{array}$ & $\begin{array}{l}\text { July } 01 \\
\text { Oct } 01 \\
\text { Jan } 02 \\
\text { April } 02 \\
\end{array}$ & see [23] & see [23] & 2004 & 2004 & 2004 & $\begin{array}{l}\text { Dec } 03 \text { to } \\
\text { Nov } 04\end{array}$ & $\begin{array}{l}1997- \\
2001\end{array}$ & $\begin{array}{l}\text { Nov } 99 \text { - } \\
\text { Nov } 00\end{array}$ & $2000-2002$ & 1998-2002 & $2000-2005$ & $\begin{array}{l}\text { Aug 03-Aug } \\
05\end{array}$ & $\begin{array}{l}1996- \\
2004\end{array}$ \\
\hline $\begin{array}{l}\text { Age } \\
\text { (years) }\end{array}$ & $\begin{array}{l}0-14: 12 \% \\
15-34: \\
50 \% \\
35-59: \\
31 \% \\
60+: 7 \%\end{array}$ & see [23] & see [23] & $\begin{array}{l}<21: 22 \% \\
21-59: 71 \% \\
>60: 4 \%\end{array}$ & $\begin{array}{l}\text { 0-14: 6\%; } \\
\text { 15-44: } \\
66 \% \\
45-64: \\
14 \% \\
65+: 4 \%\end{array}$ & $\begin{array}{l}12-24: 31 \% \\
25-34: 29 \%\end{array}$ & $\begin{array}{l}\text { 0-4: } 2 \% \\
\text { 5-14: } 8 \% \\
\text { 15-19: } 14 \% \\
\text { 20-24: } 13 \% \\
\text { 25-44: } 39 \% \\
\text { 45-64: } 19 \% \\
65+: 5 \%\end{array}$ & - & $\begin{array}{l}\text { 20-35: } 47 \% \\
\text { no specific } \\
\text { data }\end{array}$ & $\begin{array}{l}0-19: 25 \% \\
20-39: 55 \% \\
40-59: 15 \% \\
60+: 5 \%\end{array}$ & - & $\begin{array}{l}\text { 0-10: } 2 \% \\
\text { 11-20: } 7 \% \\
\text { 21-30: } 33 \% \\
\text { 31-40: } 25 \% \\
\text { 41-50: } 20 \% \\
51+: 11 \%\end{array}$ & $\begin{array}{l}\text { 0-15: } 10.3 \% \\
\text { 15-20: } 14 \% \\
\text { 21-30: } 32 \% \\
\text { 31-40: } 20 \% \\
\text { 41-50: } 11 \% \\
51-60: 5 \% \\
60+: 8 \%\end{array}$ & $\begin{array}{l}\text { Range: } \\
\text { Pr: } 14-86 \\
\text { Po:16-79 } \\
\text { Mean: } \\
\text { Pr: } 32 \\
\text { Po: } 34\end{array}$ \\
\hline $\begin{array}{l}\text { Sex ratio } \\
\text { (M:F) }\end{array}$ & $2: 1$ & $2: 1$ & $2: 1$ & 3.1:1 & $2.3: 1$ & $2.5: 1$ & $2.5: 1$ & - & $2.5: 1$ & $2.6: 1$ & - & $2.4: 1$ & 1.9:1 & $\begin{array}{l}\text { Pr:1.6:1 } \\
\text { Po: 1.9:1 }\end{array}$ \\
\hline Occupation & - & Yes & Yes & Yes & Yes & - & - & - & - & - & - & - & - & - \\
\hline $\begin{array}{l}\text { Arrived by } \\
\text { EMS }\end{array}$ & $14.4 \%$ & $14.4 \%$ & $14.4 \%$ & $29.4 \%$ & - & - & - & - & - & - & - & - & - & - \\
\hline Mechanism & Yes & Yes & Yes & Yes & Yes & Yes & Yes & Yes & Yes & Yes & Yes & Yes & Yes & Yes \\
\hline $\begin{array}{l}\text { Location of } \\
\text { injury }\end{array}$ & - & - & - & Yes & Yes & Yes & - & - & - & - & - & - & - & - \\
\hline $\begin{array}{l}\text { ISS/AIS } \\
\text { severity }\end{array}$ & - & - & - & - & - & - & - & - & - & - & - & ISS category & Deaths only & $\begin{array}{l}\text { AIS } \geq 3 \\
I S S>15\end{array}$ \\
\hline ICD & - & - & - & - & - & - & - & - & - & - & - & - & - & - \\
\hline GCS & - & - & - & - & & & - & - & - & - & - & - & - & - \\
\hline $\begin{array}{l}\text { Injury } \\
\text { description }\end{array}$ & - & - & - & - & Yes & Yes & - & - & - & - & - & - & - & - \\
\hline RTS & - & - & - & - & - & - & - & - & - & - & - & - & - & - \\
\hline TRISS & - & - & - & - & - & - & - & - & - & - & - & - & - & - \\
\hline
\end{tabular}


Table 5 Summary of key study characteristics (Continued)

\section{ICU}

admission

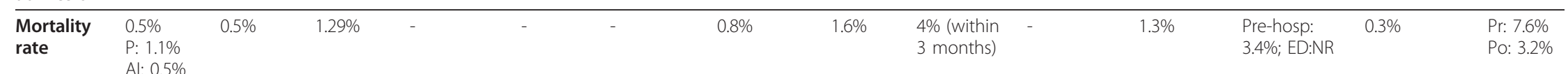

rate

16 days -

Length of

A.: $0.5 \%$

stay

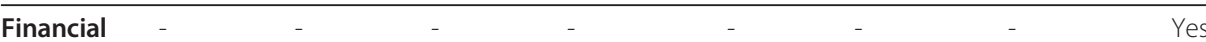

Abbreviations: RC: Reporting Card; Collab: Collaborative study; (-) indicates data not reported; Prosp: Prospective; Retro: Retrospective; Unint: Unintentional; NR: Not reported; Pr: Pre-phase of study; Po: Post-phase of study; ED: Emergency Department; P: Poisoning; Al: Acute Injury 
Table 6 Patient-focussed clinical parameters reported in the Reviews

\begin{tabular}{|c|c|c|}
\hline Parameter & $\begin{array}{l}\% \text { of studies (number of } \\
\text { references) }\end{array}$ & Comments and detail \\
\hline \multicolumn{3}{|l|}{ Patient Factors } \\
\hline $\begin{array}{l}\text { Age distribution } \\
\text { (years) }\end{array}$ & $\begin{array}{l}84 \% \\
(11 \text { of } 13)\end{array}$ & $\begin{array}{l}\text { - No uniformity of categories }[23-26,28,31,33,34] \text {, failure to report of full age } \\
\text { range }[27,30,35]\end{array}$ \\
\hline Sex distribution (M:F) & $\begin{array}{l}84 \% \\
(11 \text { of } 13)\end{array}$ & - Reported number and \% male \& females [23-28,30,31,33-35] \\
\hline Occupation & $\begin{array}{l}23 \% \\
(3 \text { of } 13)\end{array}$ & $\begin{array}{l}\text { - Transportation worker, student, farmer, technical worker, service workers } \\
\text { [24] } \\
\text { - Worker, farmer, fisher [25] } \\
\text { - Worker, student, farmer/forester/fisher [33] }\end{array}$ \\
\hline \multicolumn{3}{|l|}{ System Factors } \\
\hline Pre-hospital care & $\begin{array}{l}23 \% \\
(3 \text { of } 13)\end{array}$ & • EMS or other [23,24]; medical aid [25] \\
\hline \multicolumn{3}{|c|}{ Factors related to circumstances of injury } \\
\hline Mechanism & $100 \%$ & $\begin{array}{l}\text { - 'Super-categories' (i.e., unintentional, transport) } \\
\text { • Non-uniform use of transport/traffic }\end{array}$ \\
\hline Location & $\begin{array}{l}23 \% \\
(3 \text { of } 13)\end{array}$ & $\begin{array}{l}\text { - Industrial, road/street, home, school [25] } \\
\text { - Road/street; family, operational site [26] } \\
\text { - Operational site; road street [27] } \\
\end{array}$ \\
\hline \multicolumn{3}{|c|}{ Injury Coding and clinical indicators } \\
\hline $\begin{array}{l}\text { Abbreviated Injury Score } \\
\text { severity [35] }\end{array}$ & $\begin{array}{l}7.7 \% \\
(1 \text { of } 13)\end{array}$ & - For isolated trauma AIS $\geq 3$ [35] \\
\hline Injury Severity Score [17] & $\begin{array}{l}23 \% \\
(3 \text { of } 13)\end{array}$ & $\begin{array}{l}\text { - For those admitted to ED, ISS: < 16; 16-24 [33] } \\
\text { - For deaths only [34] } \\
\text { - For multi-trauma ISS } \geq 15 \text { [35] }\end{array}$ \\
\hline ICD [18] & None & \\
\hline 'Other injury' description & $\begin{array}{l}15 \% \\
(2 \text { of } 13)\end{array}$ & $\begin{array}{l}\text { - Superficial; open; fracture (not by region) [26] } \\
\text { - Superficial; open; fracture (not by region) [27] }\end{array}$ \\
\hline GCS [16] & None & \\
\hline RTS [19] & None & \\
\hline TRISS [20] & None & \\
\hline \multicolumn{3}{|l|}{ Outcomes } \\
\hline Mortality & $\begin{array}{l}69 \% \\
(9 \text { of } 13)\end{array}$ & $\begin{array}{l}\text { - Overall mortality plus traffic separately [29] } \\
\text { - \% within 3-months [30] } \\
\text { - Pre-hospital mortality only [33] } \\
\text { - Based on registry log only [34] } \\
\text { - Patient mortality in ED }[23,24,28,32,35]\end{array}$ \\
\hline Admission to ICU & None & \\
\hline Length of stay & $\begin{array}{l}7.7 \% \\
(1 \text { of } 13)\end{array}$ & • Mean given [28] \\
\hline Costs & $\begin{array}{l}7.7 \% \\
(1 \text { of } 13)\end{array}$ & • Costs per stay [28] \\
\hline
\end{tabular}

were identified (Table 5). The Reporting Card commenced widespread use in late 2005 as the basis of NISS, later than the publishing date of these studies. Each study collected data prospectively at three [26], six [25], 10 [27] and 26 [28] hospitals for a period of 12months, reflecting the expansion of NISS. The Reporting Card collects basic patient, mechanism, and outcomes; however the studies were mixed in the reporting of these aspects (Table 5). For instance, only one of the four reported mortality [28], one reported arrival by EMS [25], two noted occupation [25,26] and two provided a simple description of injuries sustained but without reference to body region [26,27]. The reported age categories also differed, with $\mathrm{Li}$ et al. [28] providing the most comprehensive. Notable aspects of each study are described below with detail provided in Tables 5, 6 and 7.

Zhang and Zhan [25] reported the characteristics of 1882 patients in six hospitals in the Huangdao district of Qing-dao city. 'Blunt instrument injury' (28.6\%) and traffic related injuries $(26.8 \%)$ were the two dominant injury mechanisms. The use of broad age categories 
Table 7 Leading causes of injury in the Reviewed studies, with WHO Global Burden of Disease incident cases

\begin{tabular}{|c|c|c|c|c|c|c|c|c|}
\hline 'Review' Study & Author & 1 & 2 & 3 & 4 & 5 & 6 & 7 \\
\hline \multirow{3}{*}{$\begin{array}{l}\text { '25 Emergency } \\
\text { Departments' } \\
\text { study }\end{array}$} & Chen et al [23] & Machine (29.9\%) & $\begin{array}{l}\text { Transport } \\
(24.6 \%)\end{array}$ & $\begin{array}{l}\text { Assault } \\
(17.8 \%)\end{array}$ & Falls (16.7\%) & Poisons (8.6\%) & & \\
\hline & $\begin{array}{l}\text { Li et al [24]: } \\
\text { City }\end{array}$ & Machine (32.9\%) & $\begin{array}{l}\text { Transport } \\
(21.8 \%)\end{array}$ & Falls (17.9\%) & $\begin{array}{l}\text { Assault } \\
(13.5 \%)\end{array}$ & Poisons (4.7\%) & & \\
\hline & $\begin{array}{l}\text { Li et al [24]: } \\
\text { Rural }\end{array}$ & Transport (35.3\%) & $\begin{array}{l}\text { Machine } \\
(18.2 \%)\end{array}$ & Falls (12\%) & $\begin{array}{l}\text { Poisons } \\
(7.9 \%)\end{array}$ & Assault (7.1\%) & & \\
\hline \multirow[t]{4}{*}{$\begin{array}{l}\text { NISS Reporting } \\
\text { Card studies }\end{array}$} & $\begin{array}{l}\text { Zhang \& Zhan } \\
\text { [25] }\end{array}$ & Blunt (28.6\%) & Traffic (26.8\%) & Falls (16.5\%) & $\begin{array}{l}\text { Cutting/ } \\
\text { piercing } \\
(8.9 \%)\end{array}$ & & & \\
\hline & Zhou et al [26] & Blunt (28.1\%) & $\begin{array}{l}\text { Transport } \\
(23.8 \%)\end{array}$ & Falls (18.3\%) & $\begin{array}{l}\text { Cutting/ } \\
\text { piercing } \\
(10.3 \%)\end{array}$ & Poison (6.5\%) & & \\
\hline & Xu et al [27] & Blunt (25.8\%) & Falls (25.8\%) & $\begin{array}{l}\text { Traffic } \\
(16.8 \%)\end{array}$ & $\begin{array}{l}\text { Cutting/ } \\
\text { piercing } \\
(15.6 \%)\end{array}$ & Other (6.7\%) & & \\
\hline & Li et al [28] & Transport (36\%) & Blunt (25\%) & Falls (17\%) & $\begin{array}{l}\text { Cutting/ } \\
\text { piercing } \\
(9 \%)\end{array}$ & Poisons (6.4\%) & & \\
\hline \multirow[t]{2}{*}{$\begin{array}{l}\text { Collaborative } \\
\text { studies }\end{array}$} & Li \& Wang [29] & MVA (36.1\%) & Falls (15.3\%) & $\begin{array}{l}\text { Industrial } \\
(11.9 \%)\end{array}$ & $\begin{array}{l}\text { Assault } \\
(16.8 \%)\end{array}$ & Other (25.1\%) & & \\
\hline & Li et al [30] & $\begin{array}{l}\text { Unintentional } \\
(80.8 \%)\end{array}$ & Assault (15.6\%) & $\begin{array}{l}\text { Suicide } \\
(3.6 \%)\end{array}$ & & & & \\
\hline \multirow[t]{5}{*}{$\begin{array}{l}\text { Single-centre } \\
\text { studies }\end{array}$} & Li et al [31] & Traffic (38.4\%) & Suicide (15.9\%) & $\begin{array}{l}\text { Assault } \\
(12.8 \%)\end{array}$ & Falls (12.2\%) & $\begin{array}{l}\text { Cutting/piercing } \\
(6.2 \%)\end{array}$ & & \\
\hline & Qu et al [32] & Transport (77.8\%) & Machine (9.6\%) & Falls (8.5\%) & $\begin{array}{l}\text { Cutting/ } \\
\text { piercing } \\
(1.2 \%)\end{array}$ & & & \\
\hline & Zhou et al [33] & Transport (39.2\%) & Assault (32.7\%) & $\begin{array}{l}\text { Machine } \\
12.9 \%)\end{array}$ & Burns (2.6\%) & & & \\
\hline & Yang et al [34] & $\begin{array}{l}\text { Cutting/piercing } \\
(41.4 \%)\end{array}$ & Falls (27.6\%) & $\begin{array}{l}\text { Transport } \\
(23 \%)\end{array}$ & $\begin{array}{l}\text { Machine } \\
(3.7 \%)\end{array}$ & Burns (3.1\%) & & \\
\hline & Wen et al [35] & Traffic (53.4\%) & Falls (20.9\%) & $\begin{array}{l}\text { Cutting/ } \\
\text { piercing } \\
(9.7 \%)\end{array}$ & $\begin{array}{l}\text { Assault } \\
(10.3 \%)\end{array}$ & Machine (4.5\%) & $\begin{array}{l}\text { Firearms } \\
(0.21 \%)\end{array}$ & \\
\hline \multicolumn{9}{|l|}{$\begin{array}{l}\text { Comparator } \\
\text { studies }\end{array}$} \\
\hline \multirow[t]{4}{*}{$\begin{array}{l}\text { Global Burden } \\
\text { Disease (WHO } \\
\text { Regions) [46] }\end{array}$} & The Americas & Violence (35.9\%) & $\begin{array}{l}\text { Other } \\
\text { unintentional }{ }^{\ddagger} \\
(27.8 \%)\end{array}$ & Falls (19.8\%) & $\begin{array}{l}\text { Road traffic } \\
\text { crashes } \\
(13.3 \%)\end{array}$ & Poisoning (2\%) & Fires (1\%) & $\begin{array}{l}\text { Drowning } \\
(0.1 \%)\end{array}$ \\
\hline & Europe & Falls (36.4\%) & $\begin{array}{l}\text { Other } \\
\text { unintentional } \\
(33.9 \%)\end{array}$ & $\begin{array}{l}\text { Road traffic } \\
\text { crashes } \\
(12.4 \%)\end{array}$ & $\begin{array}{l}\text { Violence } \\
(11.1 \%)\end{array}$ & Fires (3.7\%) & $\begin{array}{l}\text { Poisoning } \\
(2.3 \%)\end{array}$ & $\begin{array}{l}\text { Drowning } \\
(0.1 \%)\end{array}$ \\
\hline & $\begin{array}{l}\text { South-East Asia } \\
\text { (incl. India) }\end{array}$ & $\begin{array}{l}\text { Other } \\
\text { unintentional }{ }^{*} \\
(40.4 \%)\end{array}$ & Falls (28.4\%) & $\begin{array}{l}\text { Road traffic } \\
\text { crashes } \\
(16.9 \%)\end{array}$ & Fires (8.3\%) & Violence (4.3\%) & $\begin{array}{l}\text { Poisoning } \\
(1.6 \%)\end{array}$ & $\begin{array}{l}\text { Drowning } \\
(0.1 \%)\end{array}$ \\
\hline & $\begin{array}{l}\text { Western Pacific } \\
\text { (incl China, } \\
\text { Aust) }\end{array}$ & $\begin{array}{l}\text { Other } \\
\text { unintentional ₹ } \\
(34.9 \%)\end{array}$ & $\begin{array}{l}\text { Road traffic } \\
\text { crashes (19.2\%) }\end{array}$ & $\begin{array}{l}\text { Violence } \\
(4.7 \%)\end{array}$ & $\begin{array}{l}\text { Poisoning } \\
(2.5 \%)\end{array}$ & Fires (1.8\%) & $\begin{array}{l}\text { Drowning } \\
(0.1 \%)\end{array}$ & \\
\hline \multirow[t]{2}{*}{ Country specific } & $\begin{array}{l}\text { Australia [47] } \\
\text { (hospitalisation) }\end{array}$ & Falls (36\%) & $\begin{array}{l}\text { Transportation } \\
(14 \%)\end{array}$ & $\begin{array}{l}\text { Intentional } \\
\text { self-harm } \\
(6 \%)\end{array}$ & Assault (6\%) & $\begin{array}{l}\text { Poisoning, } \\
\text { pharmaceuticals } \\
(2 \%)\end{array}$ & $\begin{array}{l}\text { Fires, burns } \\
\text { and scalds } \\
(1 \%)\end{array}$ & \\
\hline & $\begin{array}{l}\text { United States } \\
\text { [48] } \\
\text { (hospitalisation) }\end{array}$ & $\begin{array}{l}\text { Motor vehicle, } \\
\text { cyclist, pedestrian } \\
(39.8 \%)\end{array}$ & Falls (30.2\%) & $\begin{array}{l}\text { Struck by, } \\
\text { against } \\
(6.7 \%)\end{array}$ & $\begin{array}{l}\text { Transport, } \\
\text { other (5.3\%) }\end{array}$ & Firearm (5.3\%) & $\begin{array}{l}\text { Cut/pierce } \\
(4.9 \%)\end{array}$ & $\begin{array}{l}\text { Fire/burn } \\
+ \text { flame } \\
(2 \%)\end{array}$ \\
\hline EU-region & $\begin{array}{l}\text { EU-27 [49] } \\
\text { (fatal) }\end{array}$ & $\begin{array}{l}\text { Self-inflicted } \\
(23 \%)\end{array}$ & $\begin{array}{l}\text { Road Traffic } \\
(20 \%)\end{array}$ & Falls (17\%) & $\begin{array}{l}\text { Poisoning } \\
(5 \%)\end{array}$ & Drowning (3\%) & $\begin{array}{l}\text { Interpersonal } \\
\text { violence (2\%) }\end{array}$ & Fires (2\%) \\
\hline
\end{tabular}


Table 7 Leading causes of injury in the Reviewed studies, with WHO Global Burden of Disease incident cases (Continued)

\begin{tabular}{|c|c|c|c|c|c|c|c|}
\hline $\begin{array}{l}\text { EU-27 [49], } \\
\text { hospital } \\
\text { admissions }\end{array}$ & $\begin{array}{l}\text { Home, leisure } \\
(63 \%)\end{array}$ & $\begin{array}{l}\text { Road Traffic } \\
(14 \%)\end{array}$ & Sports (8\%) & $\begin{array}{l}\text { Suicide, } \\
\text { self-harm } \\
(6 \%)\end{array}$ & $\begin{array}{l}\text { Homicide/ } \\
\text { assault (4\%) }\end{array}$ & $\begin{array}{l}\text { Workplace } \\
(4 \%)\end{array}$ & $\begin{array}{l}\text { School } \\
(1 \%)\end{array}$ \\
\hline
\end{tabular}

‡Balance of external causes in ICD10 V01-X59, Y40-Y86, Y88, Y89

used resulted in $71 \%$ falling into the single $21-59$ year age category, with $22 \%$ under 21 years and $3.6 \%$ above 60 years. The male to female ratio was $3: 1$, the highest of any of the 'collaborative studies' reported here. Occupation was reported using the terms generic 'worker' (53\%), farmer/fisherman (14.4\%) and students (11\%). Over one-third of patients were injured in an industrial environment followed by the road, the home and at school. Only $29.4 \%$ received pre-hospital medical aid, this being the only key a-priori clinical system indicator reported.

A similar pattern of injury mechanism - with the addition of poisons being reported, can be seen in the study that involved 6948 patients presenting to two Level 3 hospitals (elite) and one Level 1 hospital in the Henan Province, reported by Zhou, Zhang and Li [26]. The age group structures differed from all other papers in this Review, with 0-14 years (6\%), 15-44 years (66\%), 45-64 years $(13.9 \%)$ and $65+$ years $(6.5 \%)$ being used. The study was one of only two in the Review to report injury details however these were reported as superficial wounds $(28.7 \%)$, open wounds (25\%) and fractures (16.3\%) without reference to body region. None of the key clinical indicators of interest were reported. This study is important as the stated aim was to set up a surveillance system to guide injury prevention policy priority setting. The authors concluded that traffic management, safety programs focussed on the young, and preventative programs targeting older adults' falls in the home were critical.

In the largest study of the Reporting Card series, $\mathrm{Xu}$ et al [27] reported on 42657 patients at 10 hospitals including two Level 3 (elite) hospitals and one Level 1 hospital in each of two cities, as well as one county level hospital and one village level hospital in Guangdong Province. Blunt instrument wound was the most common mechanism (29.8\%), followed by falls (25.8\%), and then traffic crashes (16.8\%). Limited age data was reported, with only two categories noted: $12-24$ years: (31.4\%) and 25-34 years (29.3\%) (Table 5). Injury location specified as operational (industrial) site (41\%) and roads $(31 \%)$, these being different descriptors to other studies in the Review. The same injury descriptions as Zhou et al [26] with superficial wounds (35.9\%), open wounds $(33.8 \%)$ and fractures $(10.7 \%)$ were used. None of the key injury severity and outcome indicators of interest were noted. Despite this limitation, the study is important as the stated intent was to highlight the importance of surveillance systems as the basis for injury control strategies.

In the fourth of the Reporting Card studies, Li et al [28] reported on 7065 patients who presented to one of 26 hospitals in Gaocheng due to injury. Similar mechanism categories as the other studies were used, with transport (36\%) and blunt instrument (25\%) being the leading causes of injury. The reporting of age in this study was the most comprehensive all papers in the Review, particularly for those under 25 years of age. This was the only one of the four 'reporting card' studies to report mortality, with the mortality rate being $0.86 \%$. No other key indicators of injury severity or patient outcomes were noted.

\section{Collaborative studies}

Two studies were identified as being 'collaborative studies', one being a retrospective study of patients admitted to 332 hospitals in Guangdong over a 5 year period [29] and the other a prospective study at two hospitals in Shantou over a 1-year period [30] (Table 5).

$\mathrm{Li}$ and Wang's 1997-2001 retrospective study [29] is the largest reported in this Review, with nearly 1.1 million patients admitted to an emergency department due to injury. Data was collated from Reporting Forms sent by the hospitals to a central health authority. As with all of the studies, injury mechanism was documented using standard categories, these being motor vehicle crashes (36\%), unintentional falls (15.3\%), industrial accidents (11.9\%), and assault (16.8\%) (Table 7). Despite some similarity in reporting categories, the ICD system was not used. The overall mortality rate was $1.6 \%$ with $56 \%$ being traffic-related deaths. This was the only study in the Review to report mean length of stay (16 days) as well as cost of treatment. The mean cost for treatment was CNY 5442 (USD\$790) equating to approximately CNY 5.9 billion (USD $\$ 0.86 \mathrm{bn}$ ) for the presenting patients across the 5 years at the participating hospitals. The study did not report age, gender, occupation, or location of injury, nor were any of the clinical severity indicators reported.

Li et al [30] provided details of 2611 patients presenting to two hospitals in Shantou over the period of one year (Nov 1999 - Nov 2000). The authors used a survey designed specifically for the study, although as presented the data was limited to a broad description of injury 
mechanism (i.e., [un]intentional) and a single limited age category (20-35 years: $47 \%$ ). Mechanism of injury was ill-defined, with approximately $81 \%$ of patients presenting to the ED due to unspecified 'unintentional injuries', $15.6 \%$ due to assault, and $3.6 \%$ suicide. The mortality rate, noted as being within 3-months of injury, was $4 \%$. No other indices of severity, length of stay or injury information were presented.

\section{Single centre studies}

Five single centre studies were identified, with the patient sample size ranging from 5436 [34] to 13008 patients [32] with all being three or more years in duration (Table 5). Only one study was prospective in design [31], with four being retrospective reviews. All reported mechanism of injury although categories varied (Table 7), all but one [32] reported age data, and one study failed to note the sex distribution of the sample [32]. With respect to the key outcome indicators, none of the studies reported length of stay, head injury or GCS, RTS, TRISS, financial costs, or pre-hospital care; in addition, none reported patient occupation, or location. Transport was the leading cause of injury in all but one study where cutting/piercing (41\%) was the leading injury mechanism [34] (Table 7).

Li et al [31] set out to examine violence as an injury mechanism, and in doing so collected data in a prospective manner on 11472 patients in a 3 year period using a purpose designed survey. Mechanism of injury, age, and the sex distribution was described (M:F 2.6:1), however there was no data concerning key injury severity and outcome indicators. The leading mechanisms were traffic (38.4\%), suicide (15.9\%) and assault (12.8\%). Young adults (20-39) accounted for 56\% of all patients. Four age categories were used, permitting only a limited understanding of injuries experienced by young children and older adults.

The retrospective study of 13008 patients at one hospital in Hangzhou reported by Qu et al [32] used the emergency department registry log as the basis for analysis, and reported only mechanism and mortality statistics $(1.3 \%)$. In contrast to all other studies in this Review, three-quarters of the patients presented due to injury sustained in a transport-related crash, followed by machinery (9.6\%) and falls (8.5\%). Aside from these details noted above, the study presented limited patient characteristics, injury event, clinical indices and outcome variables (Table 5).

In a 5 year study published in 2006 [33], Zhou et al reported on the characteristics of 10654 patients presenting the emergency department. Of these, 361 died (3.4\%) prior to admission to the ED and 568 (5.3\%) either refused treatment or were transferred to other hospitals. This was the only study to report pre-hospital deaths however mortality of those 'admitted' to the ED was not reported. The age distribution was divided into 10 -year intervals, with those aged $20-30$ years accounting for $33 \%$ of all presentations although the age distribution was capped at $51+$ years, the lowest of any of the studies here (Table 5). The ISS was calculated for the 9725 patients admitted and treated in the ED (ISS < 15: $62 \% ; 16-24: 22 \%$; $>24: 16 \%$ ), one of only three studies in this Review to do so (Table 6).

Yang et al's [34] 2-year study of 5346 patients presenting to one hospital in Guangzhou following injury used a registry $\log$ as the data source. Mechanism was reported and while the descriptive categories were similar, the injury cause profile differed from all other studies with cutting/piercing being the most common injury mechanism (41\%) (Table 7). No information concerning patient occupation or location of injury was presented. The age categories included children and youth combined (0-15), then used deciles with the upper category being $61+$ years; none of the studies in this Review categorised older adults in detail with age being capped in the mid-60's or being $60+$ years. Reliance on the initial registry log meant that only nine deaths were recorded, with the ISS being recorded only for these patients $(0.3 \%)$, presumably due to later examination or autopsy, although this was unclear.

The patient series presented by Wen et al [35] was a pre-post comparison on the establishment of a dedicated emergency trauma department. The 'pre-period' was 1 January 1996 to 31 December 1997 with patients being assigned to a surgical department for care (i.e., usual care). The 'post-period' was 1 January 1998 to 1 January 2004 ( $75 \%$ of patients), with patients treated within a dedicated trauma department. The study captured 8271 patients, of which $53.3 \%$ (4416) were injured in road traffic crashes (Table 5, 7). Age was reported as a mean and a range, while gender, mortality and injury mechanism were also reported. The study reported AIS for patients with an isolated injury (the only study to use AIS in the Review) and ISS for multi-trauma patients. For patients in the 'pre' trauma service period $74 \%$ (1269 of 1715) had an AIS $\geq 3$ injury compared to $77 \%$ (3998 of 5192) AIS $\geq 3$ injuries in the 'post' period. For the multi-trauma patients, $69 \%$ (220 of 318) of patients in the pre-period had an ISS $>15$ in contrast to $86 \%$ (902 of 1046) of those in the 'post' period. The establishment of the trauma service resulted in a significant reduction in a range of key process and outcome indicators (Table 8). This study is important as it provides evidence that the formation of a dedicated trauma service provides superior care on these performance metrics. The ability to report these findings clearly demonstrates the value and importance of collection and analysis of registry data. In this context it is worth 
Table 8 Reductions associated with the establishment of a dedicated trauma service compared to allocation to surgical departments, derived from Wen et al [35], and 'post' trauma department benchmark metrics (in parenthesis)

\begin{tabular}{lllll}
\hline & Isolated injury & \multicolumn{3}{l}{ Multiple injuries } \\
\hline Index & Mild, AIS $<\mathbf{3}$ & Severe, AIS $\geq \mathbf{3}$ & Mild, ISS < 15 & Severe, ISS > 15 \\
\hline Time from admission to diagnosis (min) & $52 \%(34)$ & $59.7 \%(31)$ & $57 \%(36)$ & $58 \%(36)$ \\
\hline Time from admission to operation (min) & $63 \%(41)$ & $71 \%(37)$ & $64 \%(44)$ & $72 \%(37)$ \\
\hline Length of stay (days) & $48 \%(7)$ & $26 \%(14)$ & $48 \%(8)$ & $28.6 \%(15)$ \\
\hline Mortality (\%) & No deaths (0) & $63 \%(3.4)$ & $66 \%(2.8)$ & $52 \%(6.3)$ \\
\hline Complication rate (\%) & $14 \%(1.3)$ & $30 \%(8.5)$ & $23 \%(15.3)$ & $36 \%(23.7 \%)$ \\
\hline
\end{tabular}

commenting that the purpose of this study was to evaluate trauma system change rather than the surveillance nature of the other studies in this Review, and hence the greater emphasis being placed on the collection of treatment processes and clinical outcomes than in the other studies reported in this Review.

\section{Analysis of collected patient-focussed clinical parameters} The second aim of the present Review was to determine the type and breadth of patient-focussed data collected in the studies. As noted in the Method, a minimum set of data items was specified a-priori as key indicators in the assessment of the identified studies (Table 3). Further to the discussion of each study above, Table 6 shows the number of studies that reported key patient demographic, injury mechanism and location, and severity indices. While all studies reported the mechanism of injury, high-level and mixed category descriptors were used with none using ICD-10 external cause coding (Table 7). Categories such as 'transport', 'traffic', 'unintentional injury' provide only a limited understanding of the mechanism of injury and certainly the use of precise mechanism descriptions - such as pedestrian, motorcyclist, car occupant, as recommended by a range of guidelines are required to permit comparisons between studies to be made and for building a comprehensive national injury profile.

Similarly, while most studies reported the age distribution of their sample there was a lack of uniformity in the age categories used; this was described fully in the text above. There is a need for researchers to adopt the Utstein type age categories [13,14] in order to fully understand injury risk across the age spectrum in China. Two studies failed to report the patient sex, both of these being retrospective studies; these same studies reported patient age in a limited manner. Mortality was the most commonly reported severity index (69\%, 9 of 13 studies), however only one study reported pre-hospital mortality. There was little use of standard severity indices. Two studies provided an estimate of superficial, open wounds and fractures but did not differentiate body region, despite the terms 'superficial', 'open' and 'fractures' being used in the ICD. Three studies utilised the AIS-ISS system [33-35] although did so in a limited manner. Only one study reported financial cost data with the same study reporting patient length of stay, these being two inter-related outcome variables. None of the studies in the Review reported GCS [20], RTS [21], TRISS [22], ICD codes [19] or admission to ICU.

\section{Discussion}

Set amid growing calls for the establishment of injury surveillance systems in China, we conducted a review of injury surveillance research conducted the emergency departments published locally. The systematic search identified 268 research papers with an injury and medical care focus published in the period 1997 to 2007 published in Chinese; of these 13 were broad-based injury surveillance studies set in hospital emergency departments. While commentators have pointed to the need for the conduct of injury surveillance studies, it is clear that there is an established body of research that has been conducted in the field, some of which involved multiple hospitals and a large number of patients. In addition, there is also a broader body of emergency department research focussed on specific mechanisms of injury (e.g., motor vehicle), age group, or types of injury sustained not reported here.

\section{Strengths and limitations of the reviewed studies}

The studies reviewed have a number of strengths with eight of the thirteen published papers - or 8 of 12 unique studies - being collaborative studies. Seven of the 12 studies reported data collected prospectively, including all but one of the collaborative studies. The co-ordination involved in these large scale studies is noteworthy with data from a large numbers of patients collected over extended time periods.

The reporting of clinical indicators in the collaborative studies was however limited. The six single centre studies provided little additional patient information than the collaborative studies, they ranged from 5436 to 13008 patients and were conducted for periods of up to 6 years. In contrast to the collaborative studies, four of the five 
single centre studies were retrospective in nature. Also in contrast to the collaborative studies, the ISS was reported in three of the five single centre studies; however other key indices such as ICU admission, ICD coding [19], costs and details of injuries by body region were not reported. The pre-/post-trauma service study reported by Wen and colleagues [35] highlighted impressive reductions in key patient outcomes such as length of stay, mortality, complication rates and temporal factors related to care upon establishment of a dedicated trauma service, similar to findings reported previously in the US [37-42].

In all of the studies reviewed, the depth of patient injury data with respect to internationally accepted injury and trauma scoring systems was limited. Only three studies reported the ISS [18], one reported using the AIS for specific injury coding [17], and none used the ICD system to code external cause of injury, type of injury or procedures performed [19]. Furthermore, none of the studies reported the GCS [20], the RTS [21] or the TRISS [22]. The use of standardised and internationally recognised trauma severity metrics is an integral element of health system performance monitoring $[9,21,43]$ and the application of these metrics to future research studies represents a critical development need.

Additionally, injury mechanisms, age categories, mortality endpoints, and occupation were not standardised. This lack of uniformity limits the ability to make comparisons between studies and limits the use of this data in the planning of provincial and national public health initiatives and in assessing trauma system performance over time. Similarly, the ability to draw international comparisons of system performance is limited.

The quality of data collected is a limitation of a number of the studies, particularly those using the NISS reporting card, as noted by Zhou et al [26] and Li et al [44]. Both Zhou et al. and Li et al. highlighted problems of missed patients, errors, blanks or illegible items in the Reporting Cards and data entry errors. Similarly, in a pilot study for the NISS designed to examine quality control issues in the ED-based surveillance system, Xie et al [45] reported that 291 out of 1286 registered patients were 'missed' (or inadvertently excluded) by the surveillance system, and of 941 Reporting Cards 5.2\% were found to contain errors or blanks in the cards and an additional 19\% were found to have data entry errors. The studies reported in this Review provide no data to highlight the extent of these concerns.

The scale of the research conducted in these research studies is indicative of the burden of injury facing China's sizeable population. Despite the limitations in the data reported in these studies, the detail relating to injury mechanism age can provide public health specialists with sufficiently high level information to develop targeted intervention campaigns. The ability to undertake planning and quality assurance processes would however be significantly enhanced by the adoption of uniform standards in the collection and reporting of clinical data, such as the Utstein template $[13,14]$ and the ACS Guidelines [9], a need clearly highlighted by this Review.

\section{Findings relating to mechanism of injury and patient characteristics}

In the setting of provincial, national and global public health priorities, the value of comparable data across jurisdictions cannot be understated. The studies reviewed here highlight the importance of transport, falls, and industrial accidents as the most common causes of injury (Table 7). However, assaults and poisoning feature consistently in these studies. Common to all studies was the predominance of males by a ratio of 2:1. Despite little overlap in age groups between the studies, young adults consistently represented a high proportion of presentations. Mortality rates ranged from $0.5 \%$ to $8 \%$ where reported.

Table 7 provides for purposes of comparison the WHO Global Burden of Disease (GBD) 2004 incident estimates for injuries 'severe enough to require medical attention'[46]. The GBD uses ICD-10 to categories external cause of injury and while direct comparison is imperfect, some observations can be made. The rate of poisonings among the ED patients in China appears high, ranging from $4.7 \%$ to $8.6 \%$ where recorded; in contrast the GBD estimates range from $1.6 \%$ to $2.5 \%$ in the four regions shown. Within the China series, machinerelated injuries, cutting and piercing and 'blunt' injuries were prominent among the leading causes of injury. In both the China series and the GBD, transport and falls were leading causes though the order differs. Interestingly, among the 13 Chinese papers reviewed those that included suicide did not code poisons and vice versa, potentially highlighting a significant issue in coding practices.

Table 7 also shows for purposes of comparison injury causes in Australia [47], the US [48] and Europe [49]. The Australian (2005-06) data is based on administrative hospital admission datasets that use ICD-10 and codes age in five year increments; the gender ratio was 1.5:1 (male to female). The US data relates to the National Trauma Databank of 712 hospitals and includes the years 2002 to 2006; the male to female ratio was $1.87: 1$, and notably of the $1,485,098$ persons, poisonings and drowning accounted for $0.1 \%$ of patients each [48]. The US NTB uses ICD-9-CM and also ISS for all patients irrespective of injury severity. The European Union data (EU-27) relates to fatalities and hospitalisations for the period 2005 - 2007; the mortality data is based on all member states while the hospital admissions data 
(which is location specific) is assumed to be representative of all EU states. The data is coded is based on the EU Injury Database and information collected by agencies such as EuroStat, and is coded using ICD-10.

It is notable that comparisons based on mechanism using the US, Australian and EU data with the Chinese studies is relatively straightforward. Machine-related injuries, cutting and piercing and poisoning appear more prominent in the studies in China, although road traffic injuries are either the leading or second leading cause of injury across the four jurisdictions. In contrast, fall-related injuries have a lower prominence in the Chinese studies than in the US, Australia and EU regions.

The comparison presented in Table 7 demonstrates that while some comparisons can be made they are imperfect. It is also the case that within the studies in China in this Review, the transport/traffic causes cannot be disaggregated into more specific mechanisms of driver, pedestrian etc... while no detail is provided on what constitutes 'blunt' trauma. This provides further weight of evidence that the adoption of internationally recognised data collection and reporting standards in the conduct of injury surveillance research is required.

\section{Future options for ED injury surveillance research and quality assurance processes - the role of the National Injury Surveillance System and the development of Trauma Registry Systems}

In the '25' hospitals study, Chen et al [23] conclude that 'to develop a surveillance post on injuries in the Emergency Departments of general hospitals are not only necessary, urgent, but feasible.'(pp 209 and 213). Xu et al [27] make a similar point noting that surveillance systems for the basis of injury control strategies, pointing to occupational injury and transport safety as key prevention areas. Statements such as these are indicative of the increasing recognition within China of the need for the establishment of a minimum dataset for the surveillance of injury and the monitoring of trauma outcomes as a means of guiding quality improvement processes and for setting evidence-based health policy. It is also important to recognise that the development of health systems is evolutionary in nature and is a process that is contingent upon the application of resources and the availability of expertise. Undoubtedly the implementation of population-based systems and trauma registry systems is a part of this evolutionary process, the results of which are then utilised to further refine health policy and patient care.

In this context the studies conducted to date and examined by this Review could be viewed as precursors of injury surveillance and/or comprehensive trauma registry systems in China. These studies demonstrate both the operational feasibility of these systems and their value as a means of informing public health policy and practice. It is worth noting that the establishment of trauma registry systems is a relatively recent phenomenon globally; for example, the trauma registry system that captures major trauma in Victoria, Australia, was established only a decade ago in 2001 [43]. While China has developed into a leading economic power, this has also occurred only recently $[6,50]$. While a number of barriers could be suggested for reasons as to why a trauma registry has yet to be established in China - such as language and limited opportunities for training in locations that have established registry systems, it must also be recognised that there is a need to demonstrate the value of such systems which then enables, or 'unlocks' the financial resources required for their initial establishment and on-going operation. This latter point is a particularly important consideration in the context of competing development needs, which remains a feature of China at this point in time - and this is equally applicable in other low and middle income countries.

The development of the NISS [36] introduced in 2005 goes some way in addressing the need for a national injury surveillance and registry system. Notably, four of the studies reviewed here used the NISS Reporting Card as the basis for data collection. That the NISS commenced in a limited number of hospitals supports the contention that the development of population-based health data systems is progressive. The NISS now collects information on injuries from 129 hospital emergency departments from 43 counties (20 urban centres, 23 rural centres). Information collected on the Reporting Card includes simple demographics (age, occupation), injury cause information such as time and place of occurrence, causes, intention and activity when injured, as well as time of admission. The Reporting Card also collects information on severity, outcome, clinical diagnosis, and nature and site of injury although internationally recognised scoring systems such as the ICD, ISS, RTS, and TRISS are not currently used. The inclusion of these clinical indicators and severity indices would increase NISS' value immensely, however it is recognized that the necessary training for the use of these indicators is likely to be costly until a point where a collective of local 'train-the-trainers' is established.

It is clear then that the limited use of internationally accepted trauma score metrics combined with the lack of uniformity in data collection and reporting limits the ability of hospitals and relevant health departments to assess the functioning of the trauma system.

This Review further highlights the pressing need for the establishment of trauma registry systems to address this gap. While population level public health surveillance systems play a role in determining national health priorities, trauma registries represent a fundamental 
pillar of any well functioning trauma system by enabling the assessment of individual hospital performance in the treatment of the critically ill and system-wide performance through the examination of recognized Audit Filters $[43,51,52]$. Such assessments are particularly relevant in developing and expanding trauma systems [53]. Registry data has been utilized to build the evidence base that an integrated and systematic approach to trauma management is associated with a reduction in the incidence of preventable deaths, fewer complications, shorter length of stay and improved functional outcomes [37-42,54-56].

The reviewed studies demonstrate the feasibility of establishing a registry system and as Wang et al [1] note 'China has the financial resources, organisational infrastructure, and public support to rapidly apply lessons from high income countries to achieve international best-practice standards for injury prevention and control...' (p.7). China has both an opportunity and a need to establish a trauma registry system consistent with international standards of core data [9,13-16] with appropriate site specific additions to capture nuances of the health system. Inclusion of these core data points would overcome the limitations in the reporting - and hence comparability, of the studies reviewed here. In addition to performance monitoring and quality control, the ability of trauma registry data to be used to identify injury trends, evaluate public health interventions and provide the basis for capacity building in terms of academic research, educational opportunities and the conduct of clinical trials is significant.

\section{Conclusions}

This Review of Chinese-language injury surveillance studies demonstrates that a significant body of hospitalbased injury surveillance research has been undertaken in China. These studies were generally impressive in their respective sample sizes and while the majority were prospective collaborative studies, there was a lack of uniformity in reporting key data points. Moreover, none of the studies reported patient data using internationally accepted indices of injury severity. With the incidence of injury in China increasing, commentators have called for the implementation of injury surveillance systems that utilise internationally recognised coding schemes to guide population based public health priority setting. This Review supports these calls. While the recently introduced NISS goes some way in addressing the need for a population based injury surveillance system, the lack of uniformity in the reporting of patient information and the limited use of standardised severity indices in the reviewed studies highlights the need for the establishment of hospital-based trauma registry systems. While ambitious - both in terms of the financial and human resources required, the establishment of a trauma registry system and the use of recognised injury severity and outcome metrics are necessary to enable the systematic assessment the functioning of the trauma system, which then provides the means for identifying system improvements and ultimately ensuring the optimal care of the injured patient.

\section{Additional material}

Additional file 1: Table S1. Description of categories used to classify identified research papers. Table with a description of categories used to classify identified research papers, including high level subject domain, primary focus of paper, and subject categorisations.

Additional file 2: Table S2. Number and percent (\%) of articles identified by category. Table with number and percent of articles by classification category.

\section{Acknowledgements}

This study was supported by The George Institute for Global Health, Sydney Australia and Beijing China. Funding support for author Fitzharris was also derived from Monash University Injury Research Institute. The opinions, results and conclusions reported in this paper are those of the authors and are independent from the funding sources. No endorsement by the funding sources is intended or should be inferred.

\section{Author details}

${ }^{1}$ Accident Research Centre and Injury Outcomes Research Unit, Monash Injury Research Institute, Monash University, Victoria, Australia. ${ }^{2}$ Critical Care and Trauma Division, The George Institute for Global Health, Sydney, Australia. ${ }^{3}$ Research and Development, The George Institute for Global Health, Beijing, China. ${ }^{4}$ Office of the Director, The George Institute for Global Health, Beijing, China. ${ }^{5}$ Peking University Clinical Research Institute, Peking University Health Science Center, Beijing, China. ${ }^{6}$ Faculty of Medicine, University of Sydney, Sydney, Australia. ${ }^{7}$ Faculty of Medicine, University of NSW, Sydney, Australia. ${ }^{8}$ Department of Intensive Care Medicine, St George Hospital, Sydney, Australia.

\section{Authors' contributions}

All authors were involved in study conception, interpreting the results and drafting and revising the paper. MF and JY conducted the search and interpreted the original papers. All authors were involved in the design of the classification system. All authors have read and approve of the final version of the manuscript. MF and JY are the guarantors.

\section{Competing interests}

The authors declare that they have no competing interests.

Received: 22 November 2010 Accepted: 26 October 2011 Published: 26 October 2011

\section{References}

1. Wang SY, Li YH, Chi GB, Xiao SY, Ozanne-Smith J, Stevenson M, et al: Injuryrelated fatalities in China: an under recognised public health problem. Lancet 2008, 372(9651):1765-73.

2. Anon. [Health Statistics Yearbook, 2006] (In Chinese). Beijing: Ministry of Health of the Peoples Republic of China; 2007.

3. Hu G, Baker TD, Li G, Baker SP: Injury control: an opportunity for China. Inj Prev 2008, 14(2):129-30.

4. World Health Organization: Global Burden of Disease (GBD) - Death and DALY estimates for 2002 by cause for WHO Member States, Version 3 Estimates Geneva: World Health Organisation; 2004.

5. Anon. [National Report on Injury Prevention] (In Chinese). Beijing: Ministry of Health of the Peoples Republic of China; 2007.

6. The World Bank: Data Finder: China - GDP (current US\$) 2007 Washington D. C.: The World Bank Group; 2010. 
7. Hu G, Wen M, Baker TD, Baker SP: Road-traffic deaths in China, 1985-2005: threat and opportunity. Inj Prev 2008, 14(3):149-53.

8. Zhou Y, Baker TD, Rao K, Li G: Productivity losses from injury in China. Injury 2003, 9(2):124-27.

9. American College of Surgeons Committee on Trauma: Resources for Optimal Care of the Injured Patient Chicago: American College of Surgeons; 1998.

10. Fitzharris $M$, Zhong W, Myburgh J, et al: The status of trauma registry systems in Chinese hospitals. Inj Prev, Published Online First: 13 October 2011.

11. Jiang C, Driscoll P, Woodward M, Wang Z, Yates D: Trauma care in China: challenge and development. Injury 1996, 27:471-75.

12. Liberati A, Altman DG, Tetzlaff J, Mulrow C, Gøtzsche PC, et al: The PRISMA Statement for Reporting Systematic Reviews and Meta-Analyses of Studies That Evaluate Health Care Interventions: Explanation and Elaboration. PLOS Med 2009, 6(7):e1000100.

13. Dick WF, Baskett PJF: Recommendations for uniform reporting of data following major trauma - the Utstein style: A report of a Working Party of the International Trauma Anaesthesia and Critical Care Society (ITACCS). Resuscitation 1999, 42:81-100.

14. Ringdal KG, Coats TJ, Lefering R, Di Bartolomeo S, Steen PA, Røise O, Handolin L, Lossius HM, Utstein TCD expert panel: The Utstein Template for Uniform Reporting of Data following Major Trauma. A joint revision by SCANTEM, TARN, DGU-TR, and RITG. Scand I Trauma Resusc Emerg Med 2008, 16:7.

15. National Trauma Registry Consortium (Australia \& New Zealand): The National Trauma Registry (Australia \& New Zealand) Report: 2002 Herston: National Trauma Registry Consortium (Australia \& New Zealand); 2004.

16. American College of Surgeons: National Trauma Data Bank ${ }^{\circledR}$ (NTDB) Chicago, IL; 2008.

17. AAAM: The Abbreviated Injury Scale (AIS) - 1990, 1998 Update Des Plaines, IL: Association for the Advancement of Automotive Medicine (AAAM); 1998.

18. Baker S, O'Neill B, Haddon W, Long W: The Injury Severity Score: A method for describing patients with multiple injuries and evaluating emergency care. J Trauma 1974, 14:187-96.

19. World Health Organisation: International Statistical Classification of Diseases and Health Related Problems (The) ICD-10. Second edition. Geneva: World Health Organisation (WHO); 2005.

20. Teasdale G, Jennett B: Assessment of coma and impaired consciousness: A practical scale. Lancet 1974, 2:81-4.

21. Champion H, Sacco WJ, Copes W, Gann D, Gennarelli T, Flanagan M: A Revision of the Trauma Score. J Trauma 1989, 29:623-29.

22. Boyd C, Tolson M, Copes W: Evaluating Trauma Care: The TRISS Method. J Trauma 1987, 27:370-78.

23. Chen S, Zhou J, Li Z, Wu Y: [Study on injuries from emergency departments in 25 general Hospitals] (In Chinese). Chin J Epidemiol 2004, 25(3):209-13.

24. Li Z, Chen S, Zhou J, Wu Y: [The comparison on cause and treatment of injuries between two types of Emergency Department among 25 hospitals] (In Chinese). Chin J Epidemiol 2004, 25(3):214-17.

25. Zhang J, Zhan S: [A descriptive analysis on injuries among emergency department patients in Huangdao district of Qing-dao city] (In Chinese). Chin J Dis Control \& Prev 2006, 10(1):39-41.

26. Zhou G, Zhang S, Li A: [Analysis on injury surveillance of Henan Province] (In Chinese). Chin J Dis Control \& Prev 2007, 4.

27. $\mathrm{Xu} \mathrm{Y}$, Wen J, $\mathrm{Xu} \mathrm{H}$ : [Analysis on Injury Surveillance of 10 Hospital Emergency Departments in Guangdong in 2004] (In Chinese). Chin Prev Med 2006, 4:247-50.

28. Li H, Zhu Y, Sun J, et al: [An Investigation on the Clinical Injuries among Patients in Hospitals in Gaocheng City in 2004] (In Chinese). Chin J Prev Contr Chron Non-commun Dis 2005, 13(5):214-16.

29. Li L-P, Wang S: [Study on the characteristics of incidence and death among inpatients with injury in Guangdong province from 1997 to 2001] (In Chinese). Chin J Epidemiol 2003, 24(10):905-07.

30. Li L, Huang G, Luo J, et al: [Analysis of the injury surveillance in the emergency departments] (In Chinese). Chin Prev Med 2001, 2(4):257-60.

31. Li L, Cui H, Chen B, Wang S: [Analysis on the occurrence of violence among the injury surveillance program in the Emergency Department] (In Chinese). Chin J Epidemiol 2004, 25(1):12-17.

32. Qu Q, Shao Z, Wang H, et al: [Characteristics of 13,000 traumatic patients admitted to emergency department] (In Chinese). Chin J Emerg Med 2003, 12(11):776-777.
33. Zhou W, Su L, Qin W, et al: [Analysis of epidemiologic characteristics of trauma in 10654 patients in Guangzhou] (In Chinese). Chin J Emerg Med 2006, 15(9):784-86.

34. Yang D, Guo W, Tan YH, et al: [Epidemiological study on in-hospital first aid for 5346 trauma patient] (In Chinese). J Trauma Surg 2007, 9(4):334-36.

35. Wen L, Liu M, Xiong J, et al: Study of patterns of trauma care] (In Chinese). Chin J Crit Care Med 2005, 1.

36. Anon. [Manual of National Injury Surveillance System] (In Chinese). Beijing: Ministry of Health of the Peoples Republic of China; 2005.

37. Cales RH, Trunkey D: Preventable trauma deaths: A review of trauma care system development. JAMA 1985, 254:1059-63.

38. Celso B, Tepas J, Langland-Orban B, Pracht E, Papa L, Lottenberg L, et al: A systematic review and meta-analysis comparing outcome of severely injured patients treated in trauma centers following the establishment of trauma systems. J Trauma 2006, 60(2):371-78.

39. Davis MJ, Parr MJ: Trauma systems. Curr Opin Anasesthesiol 2001, 14:185-89.

40. McConnell KJ, Newgard CD, Mullins RJ, Arthur M, Hedges JR: Mortality benefit of transfer to Level I versus Level II trauma centers for headinjured patients. Health Serv Res 2005, 40(2):435-58.

41. Peitzman AB, Courcoulas AP, Stinson C, Udekwu AO, Billiar TR, Harbrecht BG: Trauma Center Maturation: Quantification of Process and Outcome. Ann Surg 1999, 230(1):87-94.

42. Trunkey DD: History and Development of Trauma Care in the United States. Clin Orthop 2000, 374:36-46.

43. Cameron PA, Gabbe BJ, McNeil JJ, Finch CF, Smith KL, Cooper DJ, et al: The Trauma Registry as a Statewide Quality Improvement Tool. J Trauma 2005, 59(6):1469-76.

44. Li H, Ju Q, Liu C, et al: [Analysis on injury surveillance of six hospitals emergency departments among urban and rural districts in Hebei province in 2007] (In Chinese). Clinical Focus 2008, 23(17):1223-26.

45. Xie T, Li D, Wu F, et al: [Quality control and function of ED based injury surveillance system] (In Chinese). Chin J Public Health 2006, 22(2):253-4.

46. World Health Organization: Regional burden of disease estimates for 2004 Incidence, WHO Regions Geneva; 2008 [http://www.who.int/healthinfo/ global_burden_disease/estimates_regional/en/index.html], (Accessed 21 July 2011).

47. AlHW, Kreisfeld R, Harrison JE: Hospital separations due to injury and poisoning 2005-06. Injury research and statistics series no. 55. Cat. no. INJCAT 131 Canberra: AlHW; 2010.

48. American College of Surgeons: National Trauma Databank 2007 Annual Report 2007 [http://www.facs.org/trauma/ntdb/pdf/ntdbannualreport2007. pdf].

49. Bauer R, Steiner M: Report Injuries in the European Union Statistics Summary 2005 - 2007, featuring the EU Injury Database (IDB) 2009 [https://webgate.ec. europa.eu/idb/].

50. Woodall P: The dragon still roars Plymouth, England: The Economist Newspaper Limited; 2009, 75, The Economist: The World in 2010.

51. American College of Surgeons Committee on Trauma: Resources for Optimal Care of the Injured Patient. Chicago: American College of Surgeons; 1993.

52. Willis C, Gabbe BJ, Cameron PA: Measuring quality in trauma care. Injury 2007, 38(5):527-37.

53. Cryer HG, Hiatt JR, Fleming AW, Gruen JP, Sterling J: Continuous use of standard process audit filters has limited value in an established trauma system. J Trauma 1996, 41(3):389-94, discussion 94-5.

54. MacKenzie EJ, Siegel JH, Shapiro S, Moody M, Smith RT: Functional recovery and medical costs of trauma: an analysis by type and severity of injury. J Trauma 1988, 28(3):281-297.

55. Tiesman H, Young T, Torner JC, McMahon M, Peek-Asa C, Fielder J: Effects of a rural trauma system on traumatic brain injuries. J Neurotrauma 2007, 24(7):1189-97.

56. Rhodes M, Aronson J, Moerkirk G, et al: Quality of life after the trauma center. J Trauma 1988, 28:931-8.

\section{Pre-publication history}

The pre-publication history for this paper can be accessed here: http://www.biomedcentral.com/1471-227X/11/18/prepub

\section{doi:10.1186/1471-227X-11-18}

Cite this article as: Fitzharris et al:: Injury in China: a systematic review of injury surveillance studies conducted in Chinese hospital emergency departments. BMC Emergency Medicine 2011 11:18. 